\title{
Research and Realization of Network Virtual Optical Laboratory
}

\author{
Hua Chen ${ }^{1, a}$ \\ ${ }^{1}$ HuBei University of Science and Technology \\ a Email: 3230499@qq.com
}

KeyWords: Network virtual optical laboratory, Virtual teaching research, Virtual laboratory realization thinking

\begin{abstract}
Nowadays, with the rapid development of network technology and computer technology, the research of network virtual optical laboratory has become a key development project in the field of relevant teaching and research. Through the network virtual optical laboratory, the real laboratory's effect can be simulated, which has a profound significance to carry on the remote teaching. This paper introduces the research and application of the network virtual optical laboratory, and analyzes the construction and working flow of the virtual laboratory, which provides a basis for the high quality of network simulation of the laboratory teaching.
\end{abstract}

\section{Introduction}

With the rapid development of computer and network technology, it is no longer a problem to realize the virtual reality simulation of network virtual environment with the support of $3 \mathrm{D}$ virtual software. In recent years, our country has a lot of research to the network virtual simulation technology and its application is very wide, for example, the research of network virtual optical laboratory is one of the more representatives. By the construction of network virtual optical laboratory, we realize the various forms of optical experimental teaching in the education system, which has a profound practical significance.

\section{Network Virtual Laboratory’s research background and significance}

Experiment is an important means of scientific research. With the rapid development of science and technology, research institutes, schools and enterprises all over the country have their own laboratories, big and small, especially Colleges of Science and Engineering, there are many professional lab building built there. However, these traditional experiments are unable to be done without lab supplies, such as the experimental devices, experimental apparatus and raw material. Only the huge original equipment cost, maintenance and management fees can bring great restrictions and obstacles to the practice in the course. At present, these traditional laboratories cannot meet the requirements of the experimenter. With the steady development of Internet technology, simulation technology and virtual reality technology, the experimental science has a new research method -- Virtual Laboratory.

Virtual Laboratory (VL) is first presented by American Professor William Wolf in 1989. By the end of 1991, NFS built " National (Science) Laboratory Cooperation Committee " Shortly thereafter, the US federal government had a huge investment to it and built the respective virtual laboratories .in astrophysics, molecular biology and oceanography.

In addition to providing technical support to experimental teaching reform, VL has its own flexibility and adaptability. By network, VL can make the experiments reach a real experiment feel 
and result anytime and anywhere, and truly "immersive" observation of experimental phenomena can be reached. For some areas lack of experimental conditions, VL provides a more convenient way of realization approach. For some dangerous and destructive experiments, VL can avoid accident injuries.

Currently, VL plays a more and more important role in scientific research, education \& training, and other fields, so the research of VL has broad application prospects. Currently, many developed countries in the world have an increasing investment to VL research. During 1998--2006, NFS provides financial aids to many VL research projects, and the maximum investment is $\$ 400$ million, which can show the importance of VL.

\section{Network Virtual Optical Laboratory's research and application}

The construction of network virtual optical laboratory is based on network technology and computer software technology. Generally speaking, it has the differences from the traditional optical laboratory. The most basic feature of VL is that it simulates the reality but is also different from the reality. VL restores the real sense of Reality Lab (RL), which can make people have an immersed sense.Based on this, the VL also has a higher flexibility and more comprehensive features than RL. The VL can achieve many experimental operation that are difficult to be carried out in the RL .For functional integrity, VL is better than RL.

After years of research and development, the VL constructed by the application of virtual simulation technology to the experimental teaching has prominent characteristics. These characteristics are also the main factors that affect the network virtual optics laboratory to be an important means of experiment teaching. First of all, the VL has a high degree of simulation, , which to a large extent, can restore the real laboratory environment. Secondly, the virtual laboratory has the perfect function, which can be able to deal with the optical experiment teaching situation in various situations and solve many difficult problems in the RL. Finally, the virtual laboratory has a good remote interactive characteristics and makes the distance experiment teaching become possible. This greatly facilitates the experimental teaching process, marking the fundamental change of teaching mode. In addition, the VL has higher innovation space than RL. Students can have more perfect conditions to try, so as to cultivate their sense of innovation, which enables students to grasp the knowledge more firmly.

The construction of the network virtual optical laboratory has been supported by many kinds of technologies, and the VL with good experimental teaching characteristics is constructed under the influence of a variety of techniques. At present, the commonly used technologies in the construction of network virtual optical laboratory include multimedia computer technology, network technology and virtual instrument technology. The computer multimedia technology and network technology are the base of all virtual simulation work, and virtual instrument technology aims at the simulation of optical laboratory. On the basis of these three kinds of technology, the construction of network virtual optical laboratory can be better realized. The network virtual optical laboratory, constructed by technology of simulation, has spectrometers, gratings, triple prisms and other instruments that are common in RL. By virtual network, the operation of instruments is improved a lot, which can make students more easily mastered these instruments’ operation.

\section{Establishment and implementation of network virtual optical laboratory}

Through the network technology, computer technology and virtual instrument technology, a perfect network virtual optics laboratory teaching system can be established. Through this system, the 
teaching of physics experiment to students can be achieved. In general, the establishment of a network of virtual optical laboratory includes the establishment of a virtual model, software programming, network interaction, the establishment of operating interactive system and so on. With the application of the teaching system as the breakthrough point, the construction and application of the network virtual optical laboratory mainly includes several aspects that are as shown in the following figure:

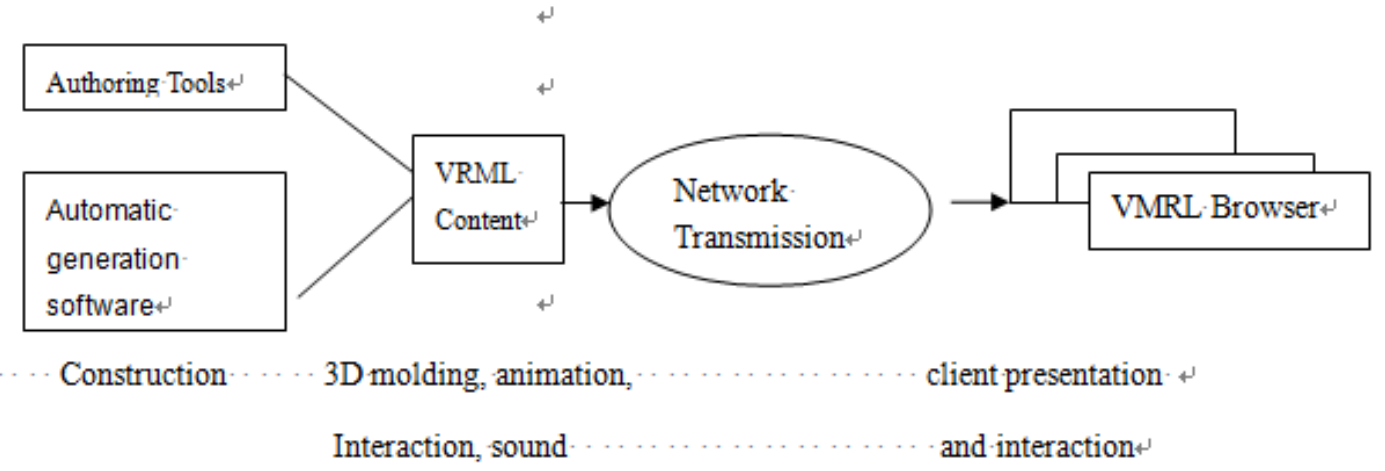

Figure 1: Implementation mode of network virtual optical laboratory

Figure 1 shows the work way of VRML system which is the core of the network virtual optical laboratory. By VRML system, network virtual laboratory can be developed, so as to achieve the goal of simulation. Generally, after the use of the VRML system, the network data transmission can be greatly reduced. Only a small amount of network data information can be needed to complete the construction of the entire network virtual optical laboratory. According to the work way of VMRL system shown in Figure 1, the realization process of the network virtual optical laboratory is as follows:

The generation of virtual model is the most basic step of constructing virtual lab, including virtual scene, virtual instrument, and other virtual facilities' generation. For example, in the optical laboratory, the more commonly used virtual instruments include triple prisms, gratings, telescopes and so on. This kind of instrument needs to be constructed virtually. In addition, the generation of virtual model also includes the generation of virtual scene, which provide the basis for restore of the realization of real experiment scene

Based on the generation of a virtual model, this kind of virtual model is inosculated into the instrument by the software. The scene, animation, sound and other content of practical optics laboratory are shown in virtual laboratory, constituting a series of virtual optical laboratory's raw data. This part also embodies VMRL system's core function, which can provide the basis for the use of laboratory teaching system for end user's .The fused comprehensive data of network virtual optical laboratory fusion are needed to be transferred to the client by network.

The show of three dimensional dynamics effects on the client

After the fused comprehensive data of network virtual optical laboratory fusion are transferred to the client by network, client software reprocesses and displays the scene, animation, sound and other information by the browser, presenting the laboratory optical scene to teachers and students. During the practical teaching, the teacher can show various types of equipment and theories by VL, and students can be also interactive with laboratory teaching system, which can deepen students' understanding of the optics knowledge.

\section{Conclusion}

In summary, network virtual optical laboratory is an important part in the current system of laboratory teaching. It provides a great help to teachers and students's laboratory teaching and 
research work. At the same time, the research and application of virtual laboratory teaching is also a key project in the development of new century teaching and an important symbol of eduction strength. With in-depth study, the simulation of network virtual optical laboratory will be highly improved. The VL will have more abundant application features and flexible response capacity, which can make the advantages of virtual laboratory in experimental teaching more clear.

\section{References:}

[1].Cai Hai-bin, Yang Yue and so on. Virtual Chemical Laboratory Based on Web, Computer Engineering and Design,2003, 24 (12): 110 112

[2]. Wang Cheng-wei, Gao Wen, Wang Hang-ren, Spirit (virtual reality) technology theory, realization and Application. Tsinghua University Press, 1996.

[3]. Jiang Xue-zhi, Li Zhong-hua. Present situation of VR researching at home and abroad, Liaoning Technical University Journal, 2004, 23(2)

[4]. Wu Wei-guo. The Design and Implementation of the Virtual Laboratory Teaching System in Middle School Physics (D), University of Electronic Science and Technology of China, 2010.

[5]. Dai Cheng-mei, Dai Cheng-jian. Design and Realization of Electrical and Electronic Network Virtual Labrory Based on LabVIEW (J). Laboratory research and exploration, 2011,02:74-77+81.

[6]. Fan Rui-ming. The Application of Virtual Reality Technology in Optical Experiment Teaching (J). Journal of Lanzhou Institute of Education, 2013,01:135-136.

[7].Ceng Qi-ming,Xue Yang, Jia Zai-yi, Mi Yang, Wang Li. The Development and Application of the Network Teaching of Modern Control Theory $(\mathrm{J})$. Laboratory research and exploration, 2013,10:146-149. 